Provided for non-commercial research and education use. Not for reproduction, distribution or commercial use.

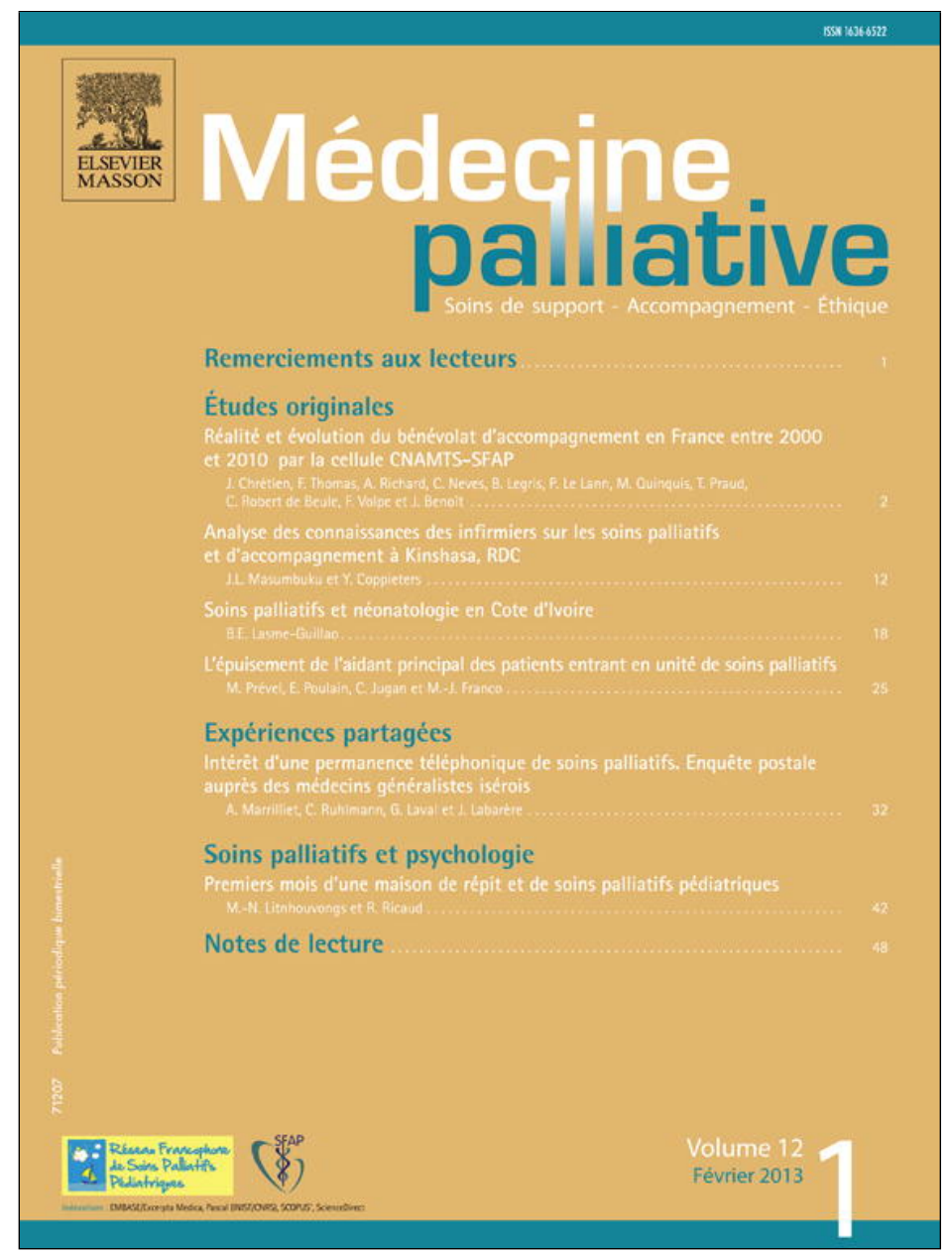

This article appeared in a journal published by Elsevier. The attached copy is furnished to the author for internal non-commercial research and education use, including for instruction at the authors institution and sharing with colleagues.

Other uses, including reproduction and distribution, or selling or licensing copies, or posting to personal, institutional or third party websites are prohibited.

In most cases authors are permitted to post their version of the article (e.g. in Word or Tex form) to their personal website or institutional repository. Authors requiring further information regarding Elsevier's archiving and manuscript policies are encouraged to visit:

http://www.elsevier.com/copyright 


\title{
Analyse des connaissances des infirmiers sur les soins palliatifs et d'accompagnement à Kinshasa, RDC
}

\author{
Analysis of nurses' knowledge on palliative care and accompaniment \\ in Kinshasa, DRC
}

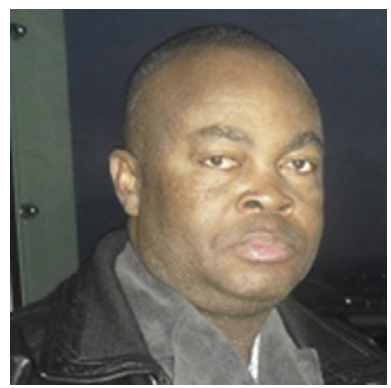

MOTS CLÉS

Connaissances ;

Infirmiers ;

Soins palliatifs ;

Kinshasa

\section{Jacques Lofandjola Masumbuku*,1, Yves Coppieters}

Centre de recherche en épidémiologie, biostatistique et recherche clinique, école de santé publique, université libre de Bruxelles (ULB), route de Lennik, 808, CP 596, 1070 Bruxelles, Belgique

Reçu le 18 janvier 2012 ; accepté le 18 mars 2012

Disponible sur Internet le 27 avril 2012

\begin{abstract}
Résumé
Contexte. - Les soins palliatifs (SP) constituent une composante de soins améliorant la qualité de fin de vie des patients. Peu de données existent sur les pratiques et connaissances des personnels soignants dans des contextes économiquement pauvres. L'objectif de cette étude est d'analyser les connaissances des infirmiers dans les hôpitaux de Kinshasa sur les soins d'accompagnement en fin de vie.

Méthodes. - Cette étude a été menée dans six hôpitaux de Kinshasa. Deux cents infirmiers ont été sélectionnés, dont 120 venant des structures publiques, 40 des structures confessionnelles et 40 des cliniques privées. Les données ont été recueillies sur base d'un questionnaire autoadministré pour analyser leurs connaissances. Étaient inclus à cette étude les infirmiers de soins intensifs, de médecine interne, de chirurgie et de pédiatrie ayant pris en charge des personnes en fin de vie.

Résultats. - Les résultats indiquent que les infirmiers (âge moyen de 43,3 $\pm 8,9$ ans, $75 \%$ de sexe féminin) enquêtés ont peu de connaissances sur les SP en fin de vie. Plus de $60 \%$ des infirmiers pensent que les SP sont équivalents à de l'acharnement thérapeutique, l'abandon du malade, les soins d'hygiène ou non adaptés.

Conclusion. - Cette étude met en évidence de connaissances insuffisantes des infirmiers sur les $\mathrm{SP}$ et d'accompagnement en fin de vie à Kinshasa. Cette étude constitue un plaidoyer au niveau des autorités compétentes pour introduire ces dimensions dans les curriculums de formation de base et continue et intégrer ces soins dans les pratiques au sein des hôpitaux de Kinshasa. (c) 2012 Publié par Elsevier Masson SAS.
\end{abstract}

\footnotetext{
* Auteur correspondant.

Adresse e-mail : jlo032000@yahoo.fr (J.L. Masumbuku).

1 Photo de l'auteur.
} 


\section{KEYWORDS}

Knowledge;

Nurses;

Palliative care;

Kinshasa

\begin{abstract}
Summary
Context. - Palliative care is a component of care, improving the quality of end-of-life of the patients. There is little data on the practices and knowledge of healthcare personnel in economically poor contexts. The aim of this study therefore is to assess what knowledge nurses in hospitals in Kinshasa have of palliative and end-of-life care.

Methods. - This study was conducted in six hospitals in Kinshasa. Two hundred nurses were selected; 120 from public establishments, 40 from denominational establishments and 40 from private clinics. The data was collected using a self-administered questionnaire to determine their knowledge. The nurses included in this study work in intensive care, internal medicine, surgery and pediatrics and have all given end-of-life care.

Results. - The results indicated that the nurses interviewed (averaging between $43.3 \pm 8.9$ years of age, $75 \%$ women) had a limited knowledge of palliative and end-of-life care. More than $60 \%$ of the nurses believe that palliative care involves either continuous treatment, abandoning the patient, personal care or inadequate care.

Conclusion. - This study highlights the fact that nurses in Kinshasa have insufficient knowledge of palliative and end of life care. This study is a plea to the competent authorities to incorporate this aspect in basic training curricula and include this field of medicine as part of the care offered in hospitals in Kinshasa.
\end{abstract}

(c) 2012 Published by Elsevier Masson SAS.

\section{Introduction}

Dans les pays industrialisés, le nombre de services de soins palliatifs (SP) et de programmes cliniques et éducationnels connaît une croissance rapide [1]. Ces programmes définissent des champs d'action avec des objectifs individuels afin de bien développer les SP. L'accès à un soutien spécialisé en cas de besoin spécifique est la réponse optimale $[2,3]$. Si les maladies non transmissibles (MNT) telles que le diabète sucré, l'hypertension artérielle, l'obésité, le cancer ont été traditionnellement considérées comme des problèmes de santé publique touchant principalement les pays développés, il est évident que ces maladies posent également des problèmes majeurs dans les pays en développement. Dans ces contextes, la fréquence des maladies transmissibles va diminuer de $3 \%$, contre une augmentation de $100 \%$ des MNT $[4,5]$. Ainsi en République démocratique du Congo (RDC), la prévalence du $\mathrm{VIH}$ est de 4,3\% [6], tandis que celle de la maladie rénale chronique est de $12 \%$ à Kinshasa [7]. Les taux de mortalité des adultes est de 6,1 pour mille chez les femmes et de 6,6 pour mille pour les hommes [6]. Face à l'émergence des MNT et la coexistence voir la réémergence des maladies transmissibles [8], il est concevable que l'application des SP en RDC puisse être quelque peu différente de celles décrites ailleurs [9]. Par conséquent, des modèles de SP adaptés pour la population congolaise sont à rechercher. Les professionnels de santé sont donc appelés à utiliser pleinement les outils mis à leur disposition afin d'aider ces personnes en fin de vie [10]. Il a été constaté que $2 \%$ seulement de programme infirmier était consacré aux soins à la fin de vie [11]. Il est visible qu'il y a un lien faible entre le curriculum d'enseignement infirmier au Congo et les SP. Cet article a comme objectif d'analyser les connaissances des infirmiers sur les SP et d'accompagnement des patients en fin de vie et de mieux comprendre les facteurs qui ne favorisent pas l'intégration de ce domaine médicale dans les pratiques infirmiers dans les hôpitaux de Kinshasa.

\section{Patients et méthodes}

Une étude descriptive transversale a été conduite auprès de 200 infirmiers (dont 150 femmes) de six formations médicales de Kinshasa:

- l'hôpital provincial général de Kinshasa;

- l'hôpital de N'djili ;

- clinique Bondeko;

- l'hôpital Saint-Joseph;

- la clinique la Candeur;

- et l'hôpital kimbanguiste.

Ces derniers ont été sélectionnés, sur base de l'existence au sein de l'institution des services de soins intensifs ou de réanimation médicale capables de prendre en charge des patients atteints des maladies chroniques en fin de vie. L'enquête a été réalisée à partir d'un questionnaire auto-administré de juin à juillet 2011. Les enquêteurs, qui étaient en majorité des prestataires de soins, ont été formés au préalable sur l'administration du questionnaire (en français). Les infirmier(e)s inclus ont été sélectionné(e)s de manière aléatoire en fonction de leur présence dans le service au moment de l'enquête. Étaient éligibles les infirmiers qui prennent régulièrement en charge des patients chroniques et les grands malades en fin de vie. Les informations recueillies, après un consentement verbal éclairé des participants, portaient sur: leurs caractéristiques sociodémographiques (âge, sexe, ancienneté et service d'affectation), le niveau de connaissance élémentaire des SP (définition tels que l'acharnement thérapeutique, l'amélioration de la qualité de vie, l'abandon des malades, les soins non adaptés, les soins d'hygiène, la prolongation de la vie, les sources de connaissance et les prestataires habilités à pratiquer cette approche médicale) et les SP proprement dits (types de soutien, les objectifs poursuivis et les apports pour les bénéficiaires). 
L'étude a reçu l'approbation du comité d'éthique de la recherche de l'Institut supérieur des techniques médicales (ISTM/Kinshasa) et des autorités des formations hospitalières étatiques, confessionnelles et privées intégrées dans l'étude.

Les données ont été encodées avec le logiciel Excel pour être ensuite analysées via Epi info version 3.3.2. Les données continues ont été rapportées avec leur moyenne et déviation standard. Le test du $\chi^{2}$ de Pearson a été appliqué pour comparer les connaissances des enquêtés en fonction de leur milieu de travail. Une $p$-value inférieure à $5 \%$ est considérée comme statistiquement significative.

\section{Résultats}

Au total 235 infirmiers ont participé à l'enquête, dont $85 \%$ ont fourni les informations complètes. Leurs caractéristiques sociodémographiques sont résumées dans le Tableau 1.

La plupart des enquêtés sont du sexe féminin (75\%) et plus de la moitié $(60 \%)$ de l'échantillon travaillaient dans les hôpitaux publics. $65 \%$ avaient une ancienneté de 11 ans et plus, $60 \%$ étaient affectés dans le service de médecine interne (Tableau 2).

La plupart des enquêtés $(98 \%)$ ont cité les médecins et infirmiers comme membres de l'équipe de SP et moins de $20 \%$ ont cité la famille, le kinésithérapeute ou prêtres (pasteur/abbé). Plus de $60 \%$ des infirmiers pensent que les SP sont équivalents à de l'acharnement thérapeutique,

Tableau 1 Caractéristiques sociodémographiques des enquêtés $(n=200)$.

Sociodemographic characteristics of surveyed $(n=200)$.

\begin{tabular}{|c|c|c|}
\hline & Moyenne $( \pm \mathrm{DS})$ & $n(\%)$ \\
\hline Âge (années) & $43,3( \pm 8,9)$ & \\
\hline \multicolumn{3}{|l|}{ Sexe } \\
\hline Masculin & & $50(25,0)$ \\
\hline Féminin & & $150(75,0)$ \\
\hline \multicolumn{3}{|l|}{ Ancienneté } \\
\hline $0-5$ ans & & $40(20,0)$ \\
\hline $6-10$ ans & & $30(15,0)$ \\
\hline$\geq 11$ ans & & $130(65,0)$ \\
\hline \multicolumn{3}{|l|}{ Lieu de travail } \\
\hline Hôpitaux publics & & $120(60,0)$ \\
\hline $\begin{array}{l}\text { Hôpitaux } \\
\text { confessionnels }\end{array}$ & & $40(20,0)$ \\
\hline Cliniques privées & & $40(20,0)$ \\
\hline \multicolumn{3}{|l|}{ Services de travail } \\
\hline $\begin{array}{l}\text { Soins intensifs et } \\
\text { réanimation }\end{array}$ & & $20(10,0)$ \\
\hline Médecine interne & & $120(60,0)$ \\
\hline Pédiatrie & & $30(15,0)$ \\
\hline Chirurgie & & $30(15,0)$ \\
\hline
\end{tabular}

DS : déviation standard; \% : pourcentage; $n$ : effectif.
Tableau 2 Éléments de connaissances de soins palliatifs sur la définition, la constitution de l'équipe, bien fondé de soins et la source d'information $(n=200)$.

Elements of knowledge of palliative care on the definition, constitution of the team, appropriateness of care and the information source $(n=200)$.

\begin{tabular}{lrr}
\hline Éléments de connaissances & \multicolumn{1}{c}{$\%$} \\
\hline Définition de soins palliatifs & & \\
Acharnement thérapeutique & 180 & 90,0 \\
Amélioration la qualité de vie & 42 & 21,0 \\
Abandon des malades & 150 & 75,0 \\
Soins non adaptés & 120 & 60,0 \\
Soins d'hygiène & 160 & 80,0 \\
Prolonger la vie & 40 & 20,0 \\
Sources de connaissances & & \\
Formation de base & 6 & 3,0 \\
Médias & 0 & 0,0 \\
Formation continue & 40 & 20,0 \\
Relations professionnelles & 4 & 2,0 \\
Constitution de l'équipe de soins palliatifs & & \\
Médecins & 194 & 97,0 \\
Infirmiers & 196 & 98,0 \\
Familles & 38 & 19,0 \\
Psychologues & 14 & 7,0 \\
Kinésithérapeutes & 38 & 19,0 \\
Prêtres (Pasteur, Abbé) & 0 & 0,0 \\
\hline
\end{tabular}

l'abandon du malade, les soins d'hygiène ou des soins non adaptés (Tableau 3 ).

Le soutien physique et spirituel a été cité par plus de la moitié des enquêtés, moins de $10 \%$ pensent que les biens fondés des SP servent à améliorer la qualité de vie, prolonger la vie et s'occuper aussi des familles. La totalité des enquêtés pense que les SP ne s'adressent qu'aux personnes âgées. Moins de $10 \%$ des enquêtés citent le psychologue comme nécessaire pour ce type de prise en charge. Aucun répondant n'a cité le domicile comme lieu d'intervention des SP et d'accompagnement et plus de $75 \%$ pensent que ces soins peuvent se faire dans le milieu hospitalier et dans les hospices des vieillards (Tableau 4).

Les connaissances des infirmiers concernant certains aspects des SP différent significativement en fonction de milieu de travail. Selon les types de soutien qu'offrent les $\mathrm{SP}$, les résultats de Tableau 4 montrent que le soutien physique et spirituel étaient cité par $75 \%$ et $91,5 \%$ des infirmiers des hôpitaux publiques, comparé aux autres, la différence était statistiquement significative $(p<0,001)$. Concernant les biens fondés des SP, le confort a été cité par $55 \%$ des infirmiers des hôpitaux confessionnels et $47 \%$ de hôpitaux publics $(p=0,005)$. Quant à la définition des SP, les résultats de ce tableau montrent une différence significative selon le milieu de travail des enquêtés. Plus de la moitié des infirmiers des hôpitaux publics et cliniques privées ont cité l'abandon et l'acharnement thérapeutique $(p<0,001)$, et $70 \%$ des infirmiers de ces mêmes structures ont soutenu que ces soins ne sont pas adaptés à Kinshasa $(p<0,001)$. En revanche, améliorer la qualité de vie était cité par $37,5 \%$ des 
Tableau 3 Éléments de connaissances des soins palliatifs sur les types de soutien, biens fondés, bénéficiaires des soins, compétences souhaitées et lieux d'intervention $(n=200)$.

Elements of knowledge of palliative care on the types of support, property based, care recipients, desired skills and intervention sites $(n=200)$.

\begin{tabular}{lrr}
\hline Éléments de connaissances & \multicolumn{2}{c}{$\%$} \\
\hline Types de soutien & & \\
$\quad$ Soutien physique & 130 & 65,0 \\
Soutien spirituel & 170 & 85,0 \\
Soutien psychologique & 4 & 2,0 \\
Soutien moral & 10 & 5,0 \\
Biens fondés de soins palliatifs & & \\
Améliorer la qualité de vie & 4 & 2,0 \\
Prolonger la vie & 10 & 5,0 \\
Confort du malade & 80 & 40,0 \\
S'occuper de la famille & 0 & 0,0 \\
Bénéficiaires des soins & & \\
Personnes âgées & 200 & 100,0 \\
Malade adulte & 60 & 30,0 \\
Enfants malades & 20 & 10,0 \\
Compétences souhaitées & & \\
Psychologues & 14 & 7,0 \\
Médecins & 194 & 97,0 \\
Kinésithérapeutes & 38 & 19,0 \\
Infirmiers & 196 & 98,0 \\
Lieu d'intervention & & \\
Domicile & 0 & 0,0 \\
Milieu hospitalier & 180 & 90,0 \\
Hospices des vieillards & 160 & 80,0 \\
\hline
\end{tabular}

infirmiers des hôpitaux confessionnels comparés aux autres $(p=0,016)$.

\section{Discussion}

L'objectif de cette étude est d'analyser les connaissances des infirmiers sur les SP et d'accompagnement des personnes en fin de vie à Kinshasa. Elle relève le niveau insuffisant des connaissances des infirmiers sur les SP et d'accompagnement dans des structures hospitalières importantes de Kinshasa. Moins de $21 \%$ des infirmiers ont dit que les SP améliorent la qualité de vie et prolongent la vie. Cette observation rejoint les résultats d'études au Lesotho et en Inde qui mettent aussi en avant le faible niveau de connaissances des infirmiers dans ce domaine [12,13]. Ces études ont montré que les infirmiers doivent améliorer leurs connaissances sur les soins d'accompagnement afin d'être en mesure de fournir des soins de meilleure qualité aux personnes malades et en fin de vie. En outre dans notre étude, $3 \%$ des infirmiers auraient entendu parler des aspects de SP dans leur formation et $20 \%$ par l'activité professionnelle à l'occasion de formations/séminaires. Ce résultat corrobore ceux de l'étude menée en Tanzanie [14]. Le manque d'éducation en SP et de programmes de formation ne favorisent pas l'administration des soins humanisés en fin de vie $[15,16]$. L'éducation formelle sur les SP est nécessaire pour fournir des soins holistiques à des patients en phase terminale [17]. Des efforts continus sont nécessaires pour surmonter les obstacles à la mise en œuvre des SP en fin de vie. Cette étude démontre que tous les infirmiers ont dit que les personnes âgées sont les bénéficiaires des SP et $10 \%$ citent de plus les enfants. Ce faible pourcentage contredit les données du terrain (exemple de la prévalence élevée de la protéinurie des enfants à Kinshasa qui est de $23,8 \%$ [18]). Il n'existe pas de programmes spécifiques de SP éducatifs à l'égard de fin de vie des enfants [19]. Ces SP sont cependant aussi nécessaires pour les enfants en fin de vie [20]. La plupart des infirmiers ont répondu à cette enquête en fonction de leurs expériences et aussi de leurs services d'affectation. La culture locale doit avoir un impact non négligeable dans la manière de répondre. Une étude antérieure réalisée aux États-Unis avait déjà rapporté que la culture fournit le contexte pour les soins de santé et les services sociaux pendant toute la vie humaine [21]. L'éducation et l'expérience clinique intégrées dans un processus continu de la qualité d'amélioration sont nécessaires pour assurer un changement durable de SP appropriés [22]. En Australie, une étude a trouvé que l'expérience des infirmiers serait à la base de leurs connaissances en SP [23]. Enfin, pour plus de $60 \%$ des infirmiers, les SP accordent le soutien physique et spirituel aux malades. Certaines études américaines ont trouvé les mêmes résultats et ont montré que les perceptions des infirmières à l'égard des personnes en fin de vie sont essentiellement centrées sur les besoins physiques [24]. Les résultats de notre étude ont mis en évidence une différence de connaissances sur certains aspects des SP selon les trois structures sanitaires. Cette différence constatée serait due à certains facteurs comme l'encadrement, la philosophie même de la dispensation des soins, l'organisation et l'accès à la formation et à l'information. À cela s'ajoute le mode de financement de chaque institution. Les hôpitaux confessionnels sont plus dans les dimensions religieuses et la charité est ancrée dans la pratique. Cette faculté va se traduire par un encadrement complet de l'infirmier. La compassion fait partie de la philosophie dans les SP [25]. Tout cela serait à la base de l'accent mis sur les soins de confort et de la qualité de vie en fin de vie par les infirmiers des hôpitaux confessionnels. Ces structures s'occupent plus de la formation et de l'information du personnel infirmier, car disposant des moyens financiers plus importants par rapport aux autres. Les hôpitaux publics au Congo sont caractérisés par la gestion laxiste et le manque de formation continue. On remarque au sein de ces hôpitaux un manque de financements. Cela pourrait justifier l'idée d'acharnement thérapeutique et d'abandon en fin de vie. Dans les cliniques privées, on note moins de préoccupations concernant l'encadrement et une sélection à l'embauche. Le goût de gain à tout prix prime sur l'intérêt du patient et du personnel. Tout ce qui précède expliquerait l'hypothèse de l'acharnement thérapeutique et l'abandon de malade en fin de vie dans ces structures.

Nos résultats plaident pour un renforcement de savoir des infirmiers par la formation et l'éducation visant l'amélioration de la qualité de vie en fin de vie. Cette étude doit favoriser une meilleure analyse des besoins de formation dans ce domaine. 
Tableau 4 Connaissances des infirmiers concernant certains aspects de soins palliatifs en fonction du milieu de travail. Knowledge of the nurses concerning certain aspects of palliative care according to workplace.

\begin{tabular}{|c|c|c|c|c|}
\hline \multirow[t]{2}{*}{ Éléments de connaissances } & \multicolumn{3}{|l|}{ Lieu de travail } & \multirow[t]{2}{*}{$p^{*}$} \\
\hline & $\mathrm{HP}(n=120)(\%)$ & $\mathrm{HC}(n=40)(\%)$ & $\mathrm{CL}(n=40)(\%)$ & \\
\hline \multicolumn{5}{|l|}{ Types de soutien } \\
\hline Soutien physique & $90(75,0)$ & $22(55,0)$ & $18(45,0)$ & $<0,001$ \\
\hline Soutien spirituel & $110(91,7)$ & $32(80,0)$ & $28(70,0)$ & 0,002 \\
\hline Soutien psychologique & $2(1,7)$ & $1(2,5)$ & $1(2,5)$ & - \\
\hline Soutien moral & $6(5,0)$ & $2(5,0)$ & $2(5,0)$ & - \\
\hline \multicolumn{5}{|l|}{ Biens fondés de SP } \\
\hline Améliorer la qualité de vie & $2(1,7)$ & $1(2,5)$ & $1(2,5)$ & - \\
\hline Prolonger la vie & $6(5,0)$ & $2(5,0)$ & $2(5,0)$ & - \\
\hline Confort du malade & $50(41,7)$ & $22(55,0)$ & $8(20,0)$ & 0,005 \\
\hline Faire participer la famille & $0(0,0)$ & $0(0,0)$ & $0(0,0)$ & - \\
\hline \multicolumn{5}{|l|}{ Bénéficiaires des soins } \\
\hline Personnes âgées & $120(100,0)$ & $40(100,0)$ & $40(100,0)$ & 1 \\
\hline Malades adultes & $30(33,3)$ & $15(37,5)$ & $15(37,5)$ & 0,167 \\
\hline Enfants malades & $10(8,3)$ & $5(12,5)$ & $5(12,5)$ & - \\
\hline \multicolumn{5}{|l|}{ Définition soins palliatifs } \\
\hline Acharnement thérapeutique & $80(66,7)$ & $15(37,5)$ & $25(62,5)$ & $<0,001$ \\
\hline Améliorer la qualité de vie & $20(16,7)$ & $15(37,5)$ & $7(17,5)$ & 0,016 \\
\hline Abandon des malades & $110(91,7)$ & $15(37,5)$ & $25(62,5)$ & $<0,001$ \\
\hline Soins non adaptés & $83(69,2)$ & $28(70,0)$ & $9(22,5)$ & $<0,001$ \\
\hline Soins d'hygiène & $100(83,3)$ & $32(80,0)$ & $28(70,0)$ & 0,189 \\
\hline Prolonger la vie & $20(16,7)$ & $10(25,0)$ & $10(25,0)$ & 0,352 \\
\hline
\end{tabular}

En dépit des limites liées à la taille de l'échantillon, nos résultats confirment un besoin réel de formation des prestataires infirmiers afin d'améliorer la prise en charge des personnes en fin de vie. Le faible niveau de connaissances serait dû à certaines contraintes comme : la méconnaissance de l'approche palliative par les professionnels de santé, la population et les autorités publiques, l'absence de formation initiale et continue des aspects relatifs aux SP. La formation sur cette approche est insuffisante et certaines croyances ou opinions négatives des infirmiers sont contraignantes.

Le système sanitaire au Congo est axé sur le modèle traditionnel de soins de santé primaires (SSP), focalisé plus dans la dimension singulière des soins curatifs selon la culture congolaise.

Lors de la revue du ministère de la Santé

Publique de RDC en février 2006, celui-ci a présenté sa nouvelle stratégie de renforcement du système de santé (SRSS). Cette stratégie prône un renforcement sur cinq axes de la zone de santé comme unité opérationnelle du système de santé. L'hôpital général de référence y devient l'élément central et une équipe cadre de la zone assure la direction de toute la zone au quotidien. De par le développement de cette politique, la RDC promulgue le renforcement de la politique des SSP en favorisant la décentralisation au niveau provincial à travers de la mise en œuvre du «Plan de Développement

Sanitaire de la Province (PDSP)» [26]. Les SP, comme une des composantes des soins globaux dans l'accompagnement en fin de vie, est quasi inexistant.

En RDC, les infirmiers de première ligne sont responsables de plus de $80 \%$ de l'offre de services. Il faut oser discuter ouvertement de la mort des grands malades en équipe pluridisciplinaire associant les familles malgré les craintes professionnelles, individuelles, familiales, culturelles et spirituelles [27].

Cette étude montre que les infirmiers à Kinshasa ont peu des connaissances sur les SP et les soins donnés en fin de vie ne sont pas humanisés. Les SP adaptés aux Africains sub-sahariens sont à rechercher et toute extrapolation des études faites en occident et en Amérique peuvent ne pas être applicables intégralement dans le contexte africain.

\section{Déclaration d'intérêts}

Les auteurs déclarent ne pas avoir de conflits d'intérêts en relation avec cet article. 


\section{Références}

[1] Clemens KE, Kumar S, Bruera E, Klaschick E, Jaspers B, De Lima L. Palliative care in developing countries: what are the important issues? Palliat Med 2007;21:173-5.

[2] Schneider N, Mitchell GK, Murray SA. Palliative care in urgent need of recognition and development in general practice: the example of Germany. BMC Fam Pract 2010;11:66.

[3] Schneider N, Lueckmann SL, Kuehne F, Klindtworth K, Behmann M. Developing targets for public health initiatives to improve palliative care. BMC Public Health 2010;10:222.

[4] Boutayeb A. The double burden of communicable and noncommunicable diseases in developing countries. Trans R Soc Trop Med Hyg 2006;100:191-9.

[5] Boutayeb A, Boutayeb S. The burden of non-communicable diseases in developing countries. Int J Equity Health 2005;4:2.

[6] Ministère du plan, RDC, Calverton, Maryland, USA: enquête démographique et de santé (EDS-RDC). 2008:p. 220-8.

[7] Sumaili EK, Krzesinski JM, Cohen EP, Nseka NM. Epidemiology of chronic kidney disease in the Democratic Republic of Congo: review of cross-sectional studies from Kinshasa, the capital. Nephrol Ther 2010;6:232-9.

[8] Tshimungu K, Okenge LN, Mukeba JN, de Mol P. Re-emergence of human African trypanosomiasis in Kinshasa, Democratic Republic of Congo (DRC). Med Mal Infect 2010;40:462-7.

[9] Sneesby L, Satchell R, Good P, van der Riet P. Death and dying in Australia: perceptions of a Sudanese community. J Adv Nurs 2011;67:2696-702.

[10] Devi PS. A timely referral to palliative care team improves quality of life. Indian J Palliat Care 2011;17:S14-6.

[11] Ferrell B, Virani R, Grant M. Analysis of end-of-life content in nursing textbooks. Oncol Nurs Forum 1999;26:869-76.

[12] Kell ME, Walley JD. Palliative care for HIV in the era of antiretroviral therapy availability: perspectives of nurses in Lesotho. BMC Palliat Care 2009;8:11.

[13] David A, Banerjee S. Effectiveness of "palliative care information booklet' ' in enhancing nurses' knowledge. Indian J Palliat Care 2011;16:164-7.

[14] Paice JA, Ferrell B, Coyle N, Coyne P, Smith T. Living and dying in East Africa: implementing the end-of-life nursing education consortium curriculum in Tanzania. Clin J Oncol Nurs 2010;14:161-6.
[15] Elcigil A. The current status of palliative care in Turkey: a nurse's perspective. J Pediatr Hematol Oncol 2011;33: S70-2.

[16] Shea J, Grossman S, Wallace M, Lange J. Assessment of advanced practice palliative care nursing competencies in nurse practitioner students: implications for the integration of ELNEC curricular modules. J Nurs Educ 2009;49:183-9.

[17] Abu-Saad Huijer H, Dimassi H, Abboud S. Perspectives on palliative care in Lebanon: knowledge, attitudes, and practices of medical and nursing specialties. Palliat Support Care 2009;7:339-47.

[18] Ekulu PM, Nseka NM, Aloni MN, Gini JL, Makulo JR, Lepira FB, et al. Prevalence of proteinuria and its association with HIV/AIDS in Congolese children living in Kinshasa, Democratic Republic of Congo. Nephrol Ther 2011. http://dx.doi.org/10.1016/j.nephr.2011.09.004.

[19] OB I, Duffy A. The developing role of children's nurses in community palliative care. Br J Nurs 2010;19:977-81.

[20] Knapp CA, Madden V, Wang H, Kassing K, Curtis C, Sloyer $P$, et al. Paediatric nurses' knowledge of palliative care in Florida: a quantitative study. Int J Palliat Nurs 2009;15: 432-9.

[21] Schim SM, Doorenbos AZ. A three-dimensional model of cultural congruence: framework for intervention. J Soc Work End Life Palliat Care 2010;6:256-70.

[22] Mahon MM, McAuley WJ. Oncology nurses' personal understandings about palliative care. Oncol Nurs Forum 2010;37:E141-50.

[23] Proctor M, Grealish L, Coates M, Sears P. Nurses' knowledge of palliative care in the Australian Capital Territory. Int J Palliat Nurs 2000;6:421-8.

[24] Meraviglia MG, McGuire C, Chesley DA. Nurses' needs for education on cancer and end-of-life care. J Contin Educ Nurs 2003;34:122-7.

[25] Kayser-Jones J, Chan J, Kris A. A model long-term care hospice unit: care, community, and compassion. Geriatr Nurs 2005;6:16-20 [p. 64].

[26] Ministère de la santé RDC. Directives d'élaboration du plan de développement de la ZS. Ministère de la Santé de RDC, juillet 2007.

[27] Fainsinger RL. Canada: palliative care and cancer pain. J Pain Symptom Manage 2002;24:173-6. 